\title{
Machine learning on field data for hydraulic fracturing design optimization
}

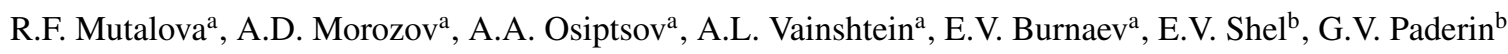 \\ ${ }^{a}$ Skolkovo Institute of Science and Technology (Skoltech), 3 Nobel Street, 143026, Moscow, Russian Federation \\ ${ }^{b}$ Gazpromneft Science $\mathcal{E}$ Technology Center, 75-79 liter D Moika River emb., St Petersburg, 190000, Russian Federation
}

\begin{abstract}
Growing amount of fracturing stimulation jobs in the recent two decades resulted in a significant amount of measured data available for construction of predictive models via machine learning (ML). Simulataneous evolution of machine learning has made it possible to apply algorithms on the hydraulic fracture database. A typical multistage fracturing job on a near-horizontal well today involves a significant number of stages. The post-fracturing production analysis (e.g., from production logging tools) reveals evidence that different stages produce very non-uniformly, and up to $30 \%$ may not be producing at all due to a combination of geomechanics and fracturing design factors. Hence, there is a significant room for fracturing design optimization, and the wealthy of field data combined with ML techniques opens a new road for solving this optimization problem. However, ML algorithms are only applicable when there is a comprehensive, well structured digital database. This paper summarizes the efforts into the creation of a digital database of field data from several thousands of multistage hydraulic fracturing jobs on near-horizontal wells from several different oilfields in Western Siberia, Russia. In terms of the number of points (fracturing jobs), the present database is a rare case of an outstandingly representative dataset of thousands of cases, compared to typical databases available in the literature, comprising tens or hundreds of pints at best. The focus is made on data gathering from various sources, data preprocessing and development of the architecture of a database as well as solving fracture design optimization via ML.
\end{abstract}

Keywords: bridging, fracture, particle transport, viscous flow, machine learning, predictive modelling, data collection, design optimization

\section{Introduction and problem formulation}

\subsection{Problem formulation in fracturing design optimization}

Hydraulic fracturing is one of the most wide-spread and commonly-used techniques for stimulation of oil and gas production from wells drilled in the hydrocarbon-bearing formation [1]. The technology is based on pumping at high pressures the fluid with proppant particles downhole through the tubing, which creates fractures in the reservoir formation via perforations. The fractures filled with granular material of closely packed proppant particles at higher-than-ambient permeability provide highly conductive channels for hydrocarbons from far field reservoir to the well all the way to surface. The technology of hydraulic fracturing is used commercially since 1947 in the US and since then the techical complexity of the stimulation treatment has made a significant step forward: wells are drilled directionally with a near-horizontal segment and multistage fractured completion.

The global aim of this study is to structure and classify existing methods and to highlight the major trends for hydraulic fracturing design optimization. Gradual development of fracturing technology is based on the advance in chemistry \& material science (fracturing fluids with programmed rheology, proppants, fibers, chemical diverters), mechanical engineering (ballactivated sliding sleeves for accurate stimulation of selected

Email address: a.osiptsov@skoltech.ru (A.A. Osiptsov) zones), and the success of fracturing stems from it being the most cost effective stimulation technique. At the same time, fracturing may be perceived as yet not fully optimized technology in terms of the ultimate production: up to $30 \%$ of fractures in a multi-stage fractured completion are not producing [2, 3]. For example, [4] analyzed distributed production logs from various stages along the near-horizontal well and concluded that almost one third of all perforation clusters are not contributing to production. The reasons for non-uniform production from various perforation clusters along horizontal wells in a plug-andperf completion are ranging from reservoir heterogeniety and geomechanics factors to fracturing design flaws. Thus, fracturing design has yet to be optimized, and it can be done either through continuum mechanics modeling (commercial fracturing simulators with optimization algorithms) or via data analytics techniques applied to a digital field database. We chose the latter route. To resolve this problem three initial classification categories are suggested: descriptive big data analytics should answer what happened during the job, predictive analytics should improve the design phase, prescriptive analytics is to mitigate production loss from non-successful jobs. Here we begin with the forward problem (predicting oil rate from hydraulic fracturing design) in order to be able to solve the inverse problem in what follows and answer the fundamental question: what is the optimum design of hydraulic fracturing job in a mulstistage fracturing completion to reach the highest possible ultimate cumulative production? 


\subsection{Recent boom in shale fracturing}

The boom in shale gas/shale oil fracturing owing to the simultaneous progress in directional drilling and multistage fracturing has resulted in extra supply in the world oil market, turning U.S. into one of the biggest suppliers. As a by-product of the shale gas technology revolution [5], there is a large amount of high-quality digital field data generated by multistage fracturing operations in shale formations of the U.S. Land, that fuel the data science research into the hydraulic fracturing design optimization [6].

Modeling of shale reservoirs is a very comprehensive problem. The flow mechanism is not always well simulated across the industry. The full scale simulation could be upgraded with ML-based pattern recognition technology where maps and history-matched production profile could enhance prediction quality for Bakken shale [7]. Marcellus shale with similar approach is detailed in [8]. Here the data driven analytics was used instead of classical hydrodynamic models.

Problem of activation of the natural fractures network by hydraulically-induced fractures is crucial for commercial production from this type of reservoirs. The mutual influence of natural and artificial fractures during the job has been studied by [9]. The research has predicted the fracture behavior when it encounters a natural fracture with the help of Artificial Neural Network (ANN) $[10,11,12]$. Similar approach is presented in [13]. Three-level index system of reservoir properties evaluation is proposed to be a new method for gas well reservoir model control in fractured reservoir based on fuzzy logic theory and multilevel gray correlation.

In [14] the authors developed a decision procedure to separate good wells from poor performers. For this purpose, the author investigated Wolfcamp well dataset. Analysis based on Decision Trees is applied to distinguish top 25 of wells from the bottom 25. Most influential subset of parameters, characterizing a well, is also selected.

\subsection{Optimization and Digitalization}

A detailed literature review on the subject is provided by [15]. They emphasized the necessity of bringing into the full scale shale gas systems a common integrating approach. They stressed the data-driven analytics to be a trend in the HF design optimization. Authors induced game-theoretic modeling and optimization methodologies to address the multiple stakeholders.

The impact of proppant pumping schedule during the job has been investigated in [16] by coupling fractured well simulator results and economical evaluations.

There are several appoaches when different target parameters are considered as criteria to optimize. For a wide range of reasons, the proppant fraction is quite an important criteria to investigate. In [17] the authors made a significant step forward gaining 4 major case studies based on shale low-permeable reservoirs across the U.S. and suggesting strategy to evaluate the realistic conductivity and impact on stimulation economics of proppant selection.
Field data, largely accumulated over the past decades, are being digitized and structured within oil companies. The disclosure of shale gas reserves linked to oil prices drop released data science slant of operators to improve the fracturing technology [18]. Increasing industry interest to artificial intelligence and to application of machine learning algorithms is justified by the coincidence of several points: processing power growth and amount of data available for analysis. Dozens of thousands of fractured wells are digitalized (e.g., see [19]), giving the grounds for the use of a wide range of big data analytics methods.

The state of affairs is a bit different in other parts of the world, where, though the wells are massively fractured, the data is not readily available and is not of that high quality as in the North America Land, which poses a known problem of "small data" analysis, where neural networks do not work, and different approaches are called for.

\subsection{Problem Statement}

Hydraulic fracturing technology is a complex process, which involves accurate planning and design using multi-discipline mathematical models based on coupled solid [20] and fluid [21] mechanics. At the same time, the comparison of flow rate prediction from reservoir simulators using fracture geometry predicted by hydraulic fracturing simulators vs. real field data suggests there is still significant uncertainty in the models.

In contrast to traditional approach to the design of hydraulic fracturing technology based on parametric studies with a hydraulic fracture simulator, we propose to investigate the problem of design optimization using ML algorithms on field data from hydraulic fracturing jobs. As a training field database, we will consider the real field data collected on fracturing jobs in Western Siberia, Russia.

The entire database from real fracturing jobs can be conventionally split into the input data and the output data. The input data in turn consists of the parameters of the reservoir and the well (permeability, porosity, hydrocarbon properties, etc.) and the job design parameters (pumping schedule). The output is basically the well flow rate after the stimulation.

The usefulness of hybrid modeling is highly reported in the literature [22]. The propagation of popular reservoir characteristics like permeability and porosity used real well-logs datasets. Nevertheless author summarizes that the suggested framework has limitation linked to the lack of human expertise.

Numerous efforts have been made by researches to implement data science to lab cost reduction issues. PVT [23] correlations correction for crude oil systems were comparatively studied between ANN and support vector machine (SVM) algorithms.

Then, the problem is formulated as follows: one may suppose that a typical hydraulically-fractured well does not reach its full potential, because the fracturing design is not optimum. Hence, a scientific question can be posed within the big data analysis discipline: what is the optimum set of fracturing design parameters, which for a given set of the reservoir characterization-well parameters yield an optimum postfracturing production? It is proposed to develop a machine 
Figure 1: Renata please make a good illustration of two pics left and right

learning algorithm, which would allow one to determine the optimum set of hydraulic fracturing design parameters based on the analysis of the reservoir-well-flow rate data.

Out of this study we expect also to be able to make recommendations on

- oil production prediction based on well information;

— the optimum frac design;

- data acquisition systems which are required to improve the quality of data analytics methods.

In the course of the study we will focus on checking the following hypotheses:

1. A methodology may be proposed for finding the optimum set of parameters to design a successful HF job.

2. Is there a problem with hydraulic fracturing design?

3. What is the objective function for optimization of HF design? What are various metrics of success?

4. How to validate the input database?

5. What database is full (sufficient)? (Optimum ratio of number of data points vs. number of features for the database?)

6. What can be learned from field data to construct a predictive model and optimize the HF design?

\subsection{Metrics of success for a fracturing job}

Optimization of a stimulation treatment is only possible if the outcome is measured. Below we summarize various approaches to quantify the success of a hydraulic fracturing job.

- Cumulative oil production of 6 and 18 months is used by [24] as a target parameter, and is predicted by a model with 18 input parameters, characterizing Bakken formation in North America.

- Predictive models for the 12 months cumulative oil production are built by [25] using multiple input parameters characterizing well location, architecture, and completions.

- Feed-forward neural network was used by [19] to predict average water production for wells drilled in Denton and Parker Counties, Texas, of the Barnett shale based on average monthly production. The mean value was evaluated using the cumulative gas produced normalized by the production time.

- In [26], a procedure was presented to optimize the fracture treatment parameters such as fracture length, volume of proppant and fluids, pump rates, etc. Cost sensitivity study upon well and fracture parameters vs NPV as a maximization criteria is used. Longer fractures does not necessarily increase NPV, a maximum discounted well revenue is observed by [27].

\begin{tabular}{|l|l|}
\hline \multicolumn{1}{|c|}{ Metrics } & Source \\
\hline $\begin{array}{l}\text { Cumulative oil production 6/18 month } \\
\text { just after the job }\end{array}$ & {$[24]$} \\
\hline 12 months cumulative oil production & {$[25]$} \\
\hline Average monthly oil production after the job & {$[19]$} \\
\hline NPV & {$[26]$} \\
\hline Comparison to modelling & {$[28]$} \\
\hline Delta of averaged Q oil & {$[29]$} \\
\hline $\begin{array}{l}\text { Pikes in liquid production for 1, 3 } \\
\text { and 12 months }\end{array}$ & {$[30]$} \\
\hline $\begin{array}{l}\text { Break even point (job cost equal to } \\
\text { total revenue after the job) }\end{array}$ & {$[32]$} \\
\hline
\end{tabular}

Table 1: Success metrics of HF job

- Statistically representative set of synthetic data served as an input for machine learning algorithm in [28]. The study analyzed the impact of each input parameter to the simulation results like cumulative gas production for contingent resources like shale gaz simulation model.

- $\Delta Q=\left(Q_{2}-Q_{1}\right)$ was an error metric to seek the re-fracture candidate for 50 wells oilfield dataset using ANN to predict after the job oil production rate $Q_{2}$ based on $Q_{1}$ oil production rate before the job [29].

- $Q$ pikes approach is presented by implementing B1, B2 and B3 statistical moving average for one, three and twelve-month best production results consequently in [30]. The simulation is done over 2000 dimension dataset to reap the benefit from proxy modeling treatment.

Net present value is one of the metrics used to evaluate the success of a hydraulic fracturing job [31]. Economical bias for hydraulic fracturing is detailed by [26]. His sequential approach of integrating upstream uncertainties to NPV creates an important tool in the identification of the crucial parameters affecting a particular job.

In Table 1, we compose a list of the main metrics for evaluation of HF job efficiency.

\subsection{Prior art in frac design and its optimization}

Typically the oilfield services industry is using numerical simulators based on the coupled solid-fluid mechanics models for evaluation and parametric analysis of the hydraulic fracturing job [20, 21, 33]. Once there is a robust forward model of the process, an optimization problem can be posed with a prescribed objective function [34].

Particular case of stimulation in carbonate reservoirs is acid frac. Iranian field with 20 fractured well has been studied by [35] in order to test candidate selection procedure. 


\subsection{Hydraulic fracture simulators for job design}

There is a variety of hydraulic fracturing simulators based on KGD, PKN, P3D, or Planar3D models of the hydraulic fracture propagation process. Shale fracturing application called for more sophisticated approaches to modeling of the fracture network propagation. A good overview of the underlying models can be found in $[20,21]$.

\subsubsection{Conventional optimization methods}

A typical approach to the optimization problem includes the construction of a surrogate of an objective function, whose evaluation involves the execution of a hydraulic fracturing simulator. The computational model integrates a hydraulic fracture simulator to predict propped fracture geometry and a production model to estimate the production flow rate. Then, an objective function is calculated, which can be any choice from Sec. 1.3 above. An example of the realization of such optimization strategy is presented in detail in [34]. Another example of an integrated multiobjective optimization workflow is given in [36], which involves a coupling of the fracture geometry module, a hydrocarbon production module and an investment-return cash flow module.

\subsubsection{ML for frac design optimization}

In North America, thanks to the great attention to multistage fracturing in shales there is an increasing amount of research papers studying the application of big data analytics to the problem of hydraulic fracturing optimization.

A general workflow of the data science approach to HF for horizontal wells implicate techniques that cluster similar critical time-series into Frac-Classes of frac data (surface treating pressure, clean and slurry pump-rates, surface and downhole amounts of mesh sand proppants placed). Correlation of the average Frac-Classes with 30-day peak production is used on the second step to distinguish between geographically distinct areas, shapes etc. [37].

Statistically representative synthetic set of data is used occasionally in the fracture model to build data-driven fracture models. The performance of the data-driven models is validated by comparing the results to a numerical model, including size, number, location, phasing angle of perforations, fluid and proppant type, rock strength, porosity, and permeability on the fracture design optimization using various fracture models. Data-driven predictive models (surrogate models, see [38]) are generated by using ANN and SVM algorithms [28].

Important geomechanics parameters are Young's modulus and Poisson's ratio obtained from laboratory wells' rock samples experiments, which is far away from covering full log heterogeneity with missing values, hence the authors used Fuzzy Logic, Functional Networks and ANNs [39].

In Russia, there are a few attempts of using ML algorithms to process data of hydraulic fracturing, e.g., the paper [32] presents the results of developing a database of 300 wells, where fracturing was performed. Operational parameters of the treatments were not taken into account in this paper. Classification models were developed to distinguish between efficient/inefficient treatments. Job success criteria were suggested in order to evaluate the impact of geological parameters on the efficiency via classification. Regression models were proposed for predicting post-frac flow rate and water cut. A portfolio of standard algorithms was used such as decision tree, random forest, boosting, ANNs, linear regression and SVM. Limitations of linear regression model applied for water cut prediction were discussed. Recent study [40] used gradient boosting to solve the regression problem for predicting the production rate after the simulation treatment on a data set of 270 wells. Mathematical model was formulated in detail, though data sources and the details of data gathering and preprocessing were not discussed.

\subsection{Our approach to the problem of hydraulic fracturing de- sign optimization using ML on field data}

An overview is made to enlight the data mining workflow and ML algorithms to petroleum engineering [41]. A robust synergy between the disciplines is required to deliver admissible prediction capability in reservoir management.

Operation-wise data management and overall response, hierarchy and time scaling is presented by [42] for decision making process across oil and gas industry.

A case with 3110 wells was investigated using ML algorithms to estimate dependence of HF from fracturing parameters. It is mentioned that heavy data mining process came before optimization - some data are considered to be erroneous and special tools like knowledge discovery and data knowledge fusion techniques [43]. The goal of the study was to identify successful practices of HF jobs.

\section{Overview of machine learning methods}

Machine learning is a broad subfield of artificial intelligence aimed to enable machines to extract patterns from data based on mathematical statistics, numerical methods, optimization, probability theory, discrete analysis, geometry, etc. Machine learning tasks are the following: classification, regression, dimensionality reduction, clustering, ranking and others. Also, machine learning is subdivided into supervised and unsupervised learning, where outputs are not labeled for the latter.

Supervised ML problem can be formulated as constructing a target function $\hat{f}: X \rightarrow Y$ approximating $f$ given a learning sample $S_{m}=\left\{\left(x_{m}, y_{m}\right)\right\}$, where $x_{m} \in X, y_{m} \in Y$ with $y_{i}=f\left(x_{i}\right)$.

To avoid overfitting (discussed in the next section), it is also very important to select ML model properly. This choice largely depends on the size, quality and nature of the data, but often without a real experiment it is very difficult to answer which of the algorithms will be really effective.

A number of the most popular algorithms such as linear models or neural networks do not cope with the lack of data, SVMs have a large list of parameters that need to be set, and trees are prone to overfitting.

In our work, we want to show how strongly the choice of the model and the choice of the initial sample can affect the final results and the correct interpretation.

Actually, there are articles with results on application of ML to HF data that describe models with high predictive accuracy. 
However, the authors use small samples with rather homogeneous data and complex models prone to overfitting. We claim that more investigations are needed, evaluating predictions accuracy and stability separately for different fields and types of wells.

\subsection{Overfitting}

Nowadays there exists an increasing trend in number of papers about application of ML in HF data processing. However, many of them might make a reader question the validity of the results, which could be erroneous due to overfitting.

Overfitting is a negative phenomenon that occurs when the learning algorithm generates a model that provides predictions mimicking a training dataset too accurately, but have very inaccurate predictions on the test data. In other words, overfitting is the use of models or procedures that violate the so-called Occam Razor [44]: the models include more terms and variables than necessary, or use more complex approaches than necessary. Figure 2 shows how the pattern of training for test and training datasets changes dramatically if overfitting takes place.
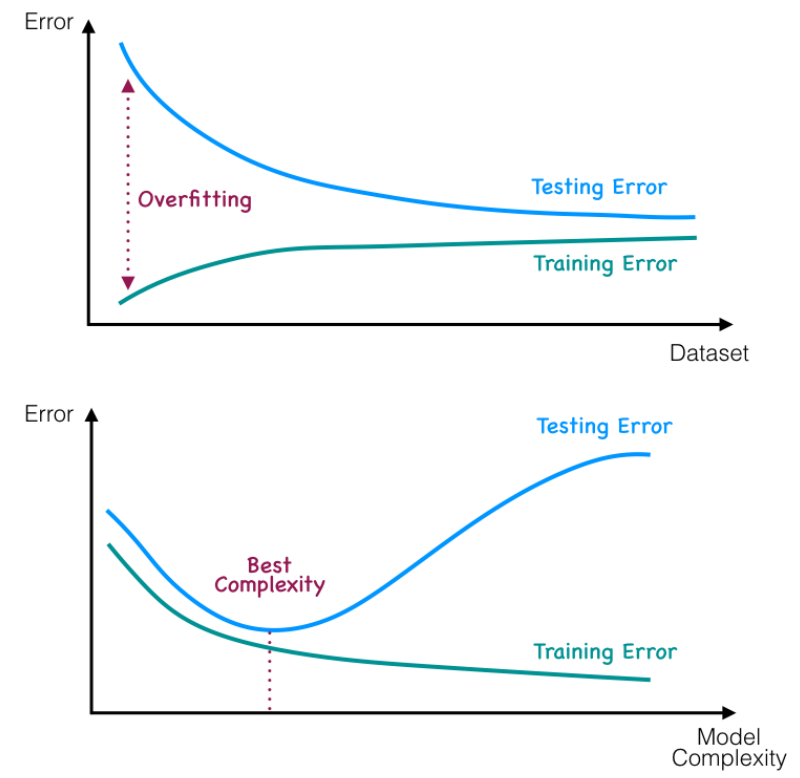

Figure 2: Overfitting

There are several reasons for this phenomenon [44, 45]:

- Traditional overfitting: learning a complex model on a small amount of data without validation. This is a fairly common problem, especially for industries that not always have access to big datasets, such as medicine, due to the complexity of data collection.

- Parameter tweak overfitting: use a learning algorithm with many parameters. Choose the parameters based on the test set performance.

- Bad statistics: misuse statistics to overstate confidence. Often some known-false assumptions about some system are made and then excessive confidence of results is derived. E.g. we use Gaussian assumption when estimating confidence.

- Incomplete prediction: use an incorrectly chosen target variable or its incorrect representation. E.g. there is a data leak and inputs already contain target variable.

- Human-loop overfitting: a human is still a part of the learning process, he/she selects hyperparameters, creates a database from measurements, so we should take into account overfitting by the entire human/computer interaction.

- Dataset selection: purposeful use of data that is well described by built models. Or use an irrelevant database to represent something completely new.

- Overfitting by review: if data can be collected from various sources, we are obliged to select only one source due to economy of resources for data collection, as well as due to computational capabilities. Thus, we consciously choose only one point of view.

For example, in the article [32] only 289 wells, each described by 178 features, were considered for the analysis. This number of points is too small compared to the number of input features, so a sufficiently complex predictive model simply "remembers" the entire dataset, but it is unlikely that the model is robust enough and can provide reliable predictions. This is also confirmed by a very large scatter of results: the coefficient of determination varies from 0.2 to 0.6 .

In this context you can find a large number of articles, where they used a disputed amount of data: e.g. in [43] - 150 wells, or in [8] - 135 wells, etc. Also, each of the mentioned articles uses a very limited choice of input features, which exclude some important stages of the hydraulic fracturing. For example, article [46] uses the following parameters to predict the quality of the hydraulic fracturing performed: stage spacing, cemented, number of stages, average proppant pumped, mass of liquid pumped, maximum treatment rate, water cut, gross thickness, oil gravity, Lower Bakken Shale TOC, Upper Bakken Shale TOC, total vertical depth. Such set of parameters does not take into account many nuances, such as the geomechanical parameters of the formation or the completion parameters of the well.

Quite good results were shown by the authors of the article [14]; they also investigated various models. But as noted in the article from 476 wells, only 171 have no $\mathrm{NaN}$ values.

In addition to the problems described above, overfitting may be caused by using too complex models: in many articles they use one of the most popular machine learning methods, the artificial neural network (ANN). But it is known that a neural network is a highly non-linear model that very poorly copes with the lack of data and is extremely prone to overfitting. Lack of data is a fairly frequent case when it comes to a real field data, which makes the use of ANNs unreliable. 
The authors of the article resort to the SVM algorithm [47]; the main disadvantage of SVM is that it has several key hyperparameters that need to be set correctly to achieve the best classification results for each given problem. The same hyperparameters can be ideal for one task and not fit at all for another. Therefore, when working with SVM a lot of experiments should be made, and the calculation takes a fairly large amount of time. Moreover, a human-loop overfitting can occur.

The above algorithms work very poorly with missing values, and so additional tricks are needed, which often leads to data leak or to various types of overfitting. Among other things these models are not easily interpretable.

In conclusion, to reduce overfitting and construct a robust predictive model, the necessary condition is to develop a big and reliable training dataset that contains all required input features.

\subsection{Dimensionality reduction}

When a problem's dataset has a large number of features (large dimension), it can cause a long ML algorithm computation time as well as difficulties in finding a good solution due to unnecessary noise in data. In addition to these reasons, a large number of features for a certain data density requires an increase in the number of samples that are economically unprofitable and risky for the oil and gas industry. At the same time, it is important to keep the completeness of information with decreasing dimension so as not to solve a meaningless task. In addition, a large dimension greatly increases the likelihood that two points are too far away, which, like outliers, leads to overfitting. Lastly, dimensionality reduction helps visualizing multidimensional data.

It should be noted that to solve our problem it is very important for us to preserve interpretability, therefore, various decompositions like PCA are not suitable.

\subsection{Clustering}

Clustering methods are used to identify groups of similar objects in a multivariate datasets. In other words, our task is to select groups of objects as close as possible to each other, which, by virtue of the similarity hypothesis, will form our clusters. The clustering belongs to the class of unsupervised learning tasks and can be used to find structures in data. Since our database includes 23 different reservoirs, horizontal and vertical wells, as well as different types of fracture design, it would be naive to assume that data is homogeneous and can be described by a single predictive model.

Thus, by dividing dataset in clusters we can obtain more homogenuous subsamples, so that ML algorithms can easily construct more accurate models on subsamples [48]. In addition, clustering is another method for detecting outliers in a multidimensional space, that can also be used for further analysis.

In our case, we used very well known k-means algorithm [49]. To evaluate quality of clustering and select optimal number of clusters ( 25 in our case) we used so-called silhouette coefficient (achieved a value of 0.71 ), which measures compactness of clusters and their separability.

\subsection{Regression}

After selecting a specific sample of data, it is necessary to solve the regression problem - to restore a continuous value from the original matrix of features. If there is a correlation $F(x)=F(x, y)$ between the variables $y$ and $x$, it becomes necessary to determine the functional relationship between the two quantities. The dependence of the mean value $\mu=f(x)$ is called the regression of $y$ over $x$.

In the reviewed articles other authors considered different approaches how to define a target variable. In particular, they considered cumulative production for 3, 6 and 12 months. However, we noted a strong corellation between values of cumulative production for 3, 6 and 12 months. Thus we as a target variable we consider only values of cumulative production for 12 months.

After building a regression model we assess its accuracy on a separate test sample. As a prediction accuracy measure we use the coefficient of determination. The coefficient of determination $\left(R^{2}-R\right.$-square) is the fraction of the variance of the dependent variable explained by the model in question.

\subsection{Ensemble of models}

The ensemble of methods uses several training algorithms in order to obtain better prediction efficiency than could be obtained from each training algorithm individually.

Ensembles, due to their high flexibility, are very prone to overfitting, but in practice, some assembly techniques, such as bagging, tend to reduce overfitting. The ensemble method is a more powerful tool compared to stand-alone forecasting models, since it minimizes the influence of randomness, averaging the errors of each basic model and reduces the variance.

\subsection{Feature importance}

The use of tree-based models makes it easy to identify features that are of zero importance, because they are not used when working. Thus, it is possible to gradually discard unnecessary features, until the calculation time and the quality of the prediction becomes acceptable, while the database does not lose its information content too much.

There is the Boruta method which is a test of the built-in solutions for finding important parameters. The essence of the algorithm is that features are deleted that have a Z-measure less than the maximum Z-measure among the added features at each iteration. Also, the Sobol method is widely used for feature importance. The method is based on the representation of the function of many parameters as the sum of functions of a smaller number of variables with special properties.

\subsection{Hyperparameter search}

Hyperparameter optimization is the problem of choosing a set of optimal hyperparameters for a learning algorithm. Whether the algorithm is suitable for the data directly depends on hyperparameters, whether there is any overfitting or underfitting. Each model requires different assumptions, weights or training speeds for different types of data under the conditions of a given loss function. 
The most common method for optimizing hyperparameters is grid search, which simply does a full search on a manually specified subset of the hyperparameter space of the training algorithm. But before using grid search, a random search was used to estimate the boundaries of a significant change in the parameters. Moreover, according to the Vapnik-Chervonenkis theory, the more flexible a model is, the worse its generalizing ability. Therefore, it is very important to stop and not to fit the model specifically to the existing database, otherwise new data will be predicted incorrectly. To check the operation and generalize the optimization of hyperparameters, a cross validation can be used.

\subsection{Validation}

Evaluation of the quality of the resulting model is the most important part of the work. The model can give very good results, but in real life can be not applicable to new test data at all. Proper construction of the training and test samples helps to provide their coinciding distributions and further to ensure the constructed predictive model to be more stable. Since the data points also have time dimension and we can make only causal predctions (we can not train the model on a "future" data and apply it to a "past" data), then for train/test data split we used the "TimeSeriesSplit" function from [49] library.

\subsection{Uncertainty Quantification}

Uncertainty comes from errors made by a ML algorithm and from noise from a dataset. Hence, predicting an output only is not sufficient to be certain with results. In terms of machine learning, quantifying uncertainty is predicting a single point that encompasses the uncertainty of that prediction. In order to achieve so, prediction intervals can be implemented which provide probabilistic upper and lower bounds on the estimate of an outcome variable.

A prediction interval is calculated as some combination of the estimated variance of the model and the variance of the outcome variable [50]. To build prediction interval for the model, the bootstrap resampling method can be used, but are computationally expensive to calculate.

Uncertainty quantification can be applied as confidence interval instead of prediction interval, which is a range of values so defined that there is a specified probability that the value of a parameter lies within it. At this way, machine learning algorithm performance's uncertainty can be estimated. Hence, there is the difference between prediction interval and confidence interval. A confidence interval quantifies the uncertainty on an estimated population variable, such as the mean or standard deviation. Whereas a prediction interval quantifies the uncertainty on a single observation estimated from the population [51].

\subsection{Reinforcement learning}

Design optimization is an inverse problem, where optimal fracturing parameters have to be found to maximize oil production. In order to solve the problem, non-gradient optimization techniques are required. Also, parameters like PVT and geomechanical properties which cannot be changed by a field engineer have to be taken into account. In addition, the problem has many parameters to optimize, and this creates a big search space, which may take a lot of time.

To deal with these issues, application of reinforcement learning is applied. Reinforcement learning refers to goal-oriented algorithm where a model/agent tries to maximize a reward by acting in a complex environment with a high level of uncertainty. In case of the current problem, it imitates field engineer's decisions in choosing optimal parameters in particular conditions based on his or her experience. Such algorithms are able to take into account an environment, and solve problems hedonistically by obtaining reward as much as possible. Importantly, reinforcement learning solves a complex problem of correlating immediate actions with the delayed returns they produce. It operates in a delayed return environment, where it can be difficult to understand which action leads to which outcome over many time steps.

In terms of reinforcement learning terminology, it has the following components:

- Strategy - determines the behavior of the learning agent at any given moment in time. It maps states to actions, the actions that promise the highest reward.;

- Incentive/reward function - associates with each perceived state of the environment a reward showing the degree of desirability of this state;

- Value function is the long-term total amount of the reward that the agent expects to receive in the future. In other words, value is the prediction of reward.

\section{Field database: structure, sources, pre-processing, sta- tistical properties, data mining}

Following the report by McKinsey\&Company from 2016 the majority of companies get real profit from annually collected data and analytics [52]. However, the main problem companies usually face while getting profit from data lies inside the organizational part of the work.

Most of the researches skip the phase of data mining, considering the ready-made dataset as a starting point for ML. Nevertheless, we can get misleading conclusions from false ML predictions due to learning on the low-quality dataset. As follows from results of [53] the most important thing when doing the ML study is not only a representative sample of the wells, but also a complete set of parameters that can fully describe the fracture process with the required accuracy.

As can be seen from Section ??, where we describe various types of overfitting, the most important one is related to a poor quality of the training dataset. In addition, if in case of a nonrepresentative training dataset we use a subsample of it to train the model, corresponding results will be very non-stable and will hide the actual state of things.

It is known that data preprocessing actually takes up to $74 \%$ of the entire time in every project [54]. Having a good, highquality and validated database is the main key to obtain the interpretable solution using ML. The database must include all 


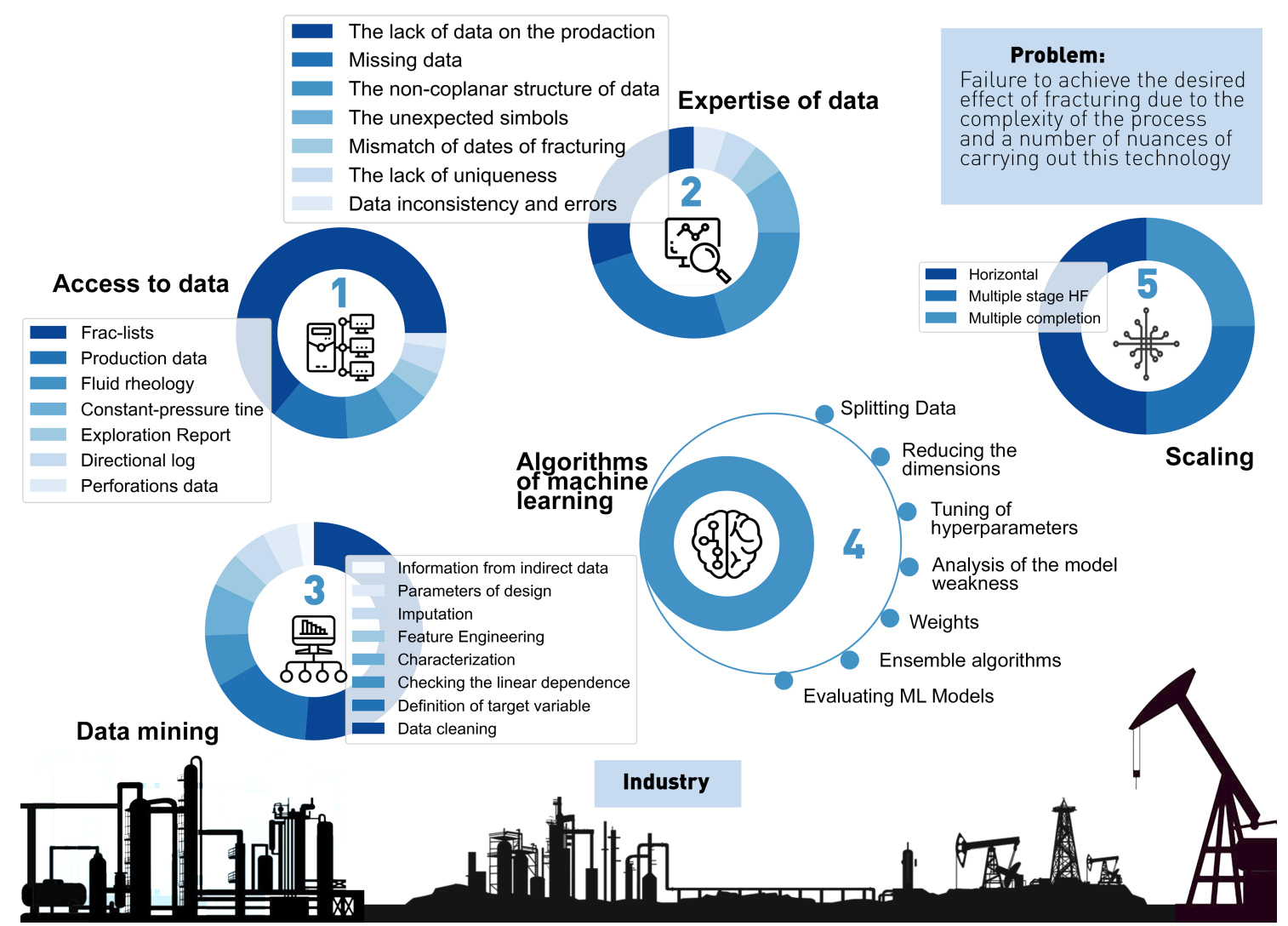

Figure 3: General workflow

the parameters that are important from the point of view of the physics of the process, be accurate in its representation and verified by subject domain experts in order to avoid the influence of errors in database maintenance.

Unfortunately, in field conditions each block of information about different stages of the hydraulic fracturing is recorded in a separate database; as a result of this work there is no integrated database containing information about sufficient number of wells that would include all factors for decision making. So, a right way to pre-process data should be done to make it more useful, work - more efficient, results - more reliable.

In the next subsections we consider stages of database preparation.

\subsection{Collecting database}

To collect all necessary information we studied the following sources: Frac-list - a document with a general description of the process and the main stages of loading; MER - a table with production collected monthly after the final commissioning; Technical regimes - geological and technical data; Geomechanical reports - stress contrasts, Poisson's ratio, strain modules for formations; RIGIS - formation data, as well as PVT-file as a general physical characteristic of the reservoir.

\subsection{Matching database}

When matching data from different sources, there is often a lack of a uniform template for different databases. To overcome this obstacle, we used regular expression algorithms and morphological analysis to identify typos. This approach allowed us to automate the process of data preparation and to make it rigorous.

To isolate typos that are inevitable in large databases, which are filled by a big number of individuals, we used created "dictionaries" for all sorts of categorical variables. With the help of the Levenshtein distance [55] we found words analogs that were considered equal. Since the "dictionary" we used was not very large, we applied the brute-force search strategy, which showed high efficiency.

\subsection{Rounding/Averaging database}

A large number of different sources often have not only different measurement systems, but also different accuracies. Many geomechanical parameters, measured by logging, represent a range of values, while sensor readings show only the average value for the entire layer.

We need accurate measurements that could help to identify characteristics of layers and transfer them to the interlayers. To make this transition from layers to interlayers, we used "dictionaries" of layers, where each layer name included a subset of interlayers with their parameters. Thus, a system was created in which the accuracy for the entire database was determined in such a way as not to average all the parameters at once, but to be able to move to a more accurate measurement and work with interlayers. 


\subsection{Database cleanup}

Unfortunately, although for categorical features we can mainly restore actual values in case of typos, this is not always the case in case of real-valued features. Also very sensitive sensors can show several values (or range of values) for a certain period of changes, and all of them are recorded as a characteristic for a given period (for example, 1000-2000). But to apply ML one unique value for each parameter is needed.

As a result we delete erroneous and uncharacteristic values: instead of values that were informational noise (e.g. a string value in a numeric parameter) the value $\mathrm{NaN}$ was used. For features values, initially represented by ranges, corresponding average values were used in order to make them unambiguous and at the same time not to add extra noise to the dataset.

\subsection{Filling missing values}

One of the main problems with working with any data is its absence. The data may simply be absent or contain too implausible values, which is most effectively replaced by a pass. In addition to this, there are a number of different powerful approaches in machine learning like the SVM regression based approach or neural networks, which require filling all the values.

To solve this problem in ML, there are a number of tricks that allow you to fill in the missing values. But it should also be noted that most approaches can be overly artificial and not correspond to reality.

Therefore, we used the method of filling NaNs with the average value using clustering, where the average of the feature was taken not from the entire sample, but from the cluster, which allows us to more accurately estimate the missing value.

\subsection{Categorical features}

Let us describe how we work with categorical features. In the entire database the number of categorical features is equal to 22. If we use one-hot encoding [56] for each unique value of each input categorical feature, the feature space is expanded with 3188 additional binary features. This leads us to the curse of dimensionality problem [57]. And obviously, increases the calculation time and risk of overfitting. Therefore, for categorical features, which usually denote the name of the proppant for hydraulic fracturing, we left the main name of the manufacturer and the number of the stage in which this substance was used. This approach allows you to indirectly save the name and size of the proppant. Thus, the binary space dimension of categorical features increases only up to 257 . Thanks to this trick we increased the calculation speed of ML model training and improved overall prediction accuracy when investigating the developed dataset.

\subsection{Linear dependence}

After encoding the categorical features, despite reduction of their dependence, the size of the feature space is still large. Linearly dependent features strongly influence the stability of predictive models, therefore, in case of such high-dimensional feature space we need to remove all dependant features. For that we used Pearson correlation [58] to find linearly dependant variables.

The final database consists of data that fully describe the situation occurring in the field and contains: the geomechanical parameters of the reservoir used, the well completion parameters, the parameters of the used chemical reagents and hydraulic fracturing fluids, the physical parameters of the hydraulic fracture and the accumulated debits that we studied for 3, 6 and 12 months. The geological data provides the information about the reservoir productivity from the well. It includes thickness of the zones in the reservoir model, average porosity, average clay content and density.

We use the developed dataset to construct an ML predictive model: we predict production of a new well based on its history, environmental condition and design parameters.

In terms of dimensions, initially we had 7009 wells with more than 200 parameters. Then, it was reduced to 5247 because wells which had hydraulic fracturing in 2012 were excluded. Finally, more than 2000 wells were excluded as outliers while around 80 parameters were removed since they were referential for other features like "executive contractor". Final dataset contains data 3110 wells, each characterized by 113 features. A Table 2 with a list of main features can be found in Appendix.

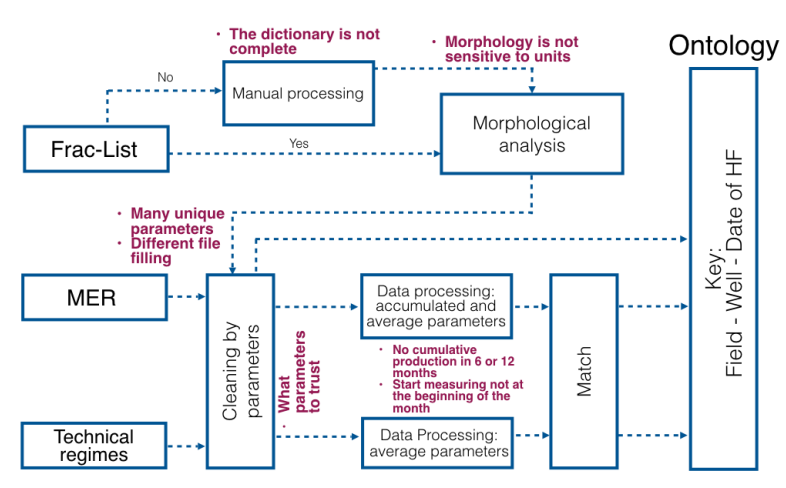

Figure 4: Block diagram (1) of the database creation

\subsection{Handling outliers}

Objects that are very different from most points often bring noise to the algorithm. Also, these are often simply not physical values that contradict the original experiment. The main types of outliers can be classified into three types: data errors (measurement inaccuracies, rounding, incorrect records), which are especially often the case with field data; the presence of noise objects, suspiciously good or bad wells, the presence of objects of other samples, the difference in field geology is too big.

To effectively detect such values, we used several techniques. These were statistical methods, when it was possible to look at the distribution in the dimension of the attribute itself and practically to see the anomalous values using the Kurtosis measure. 


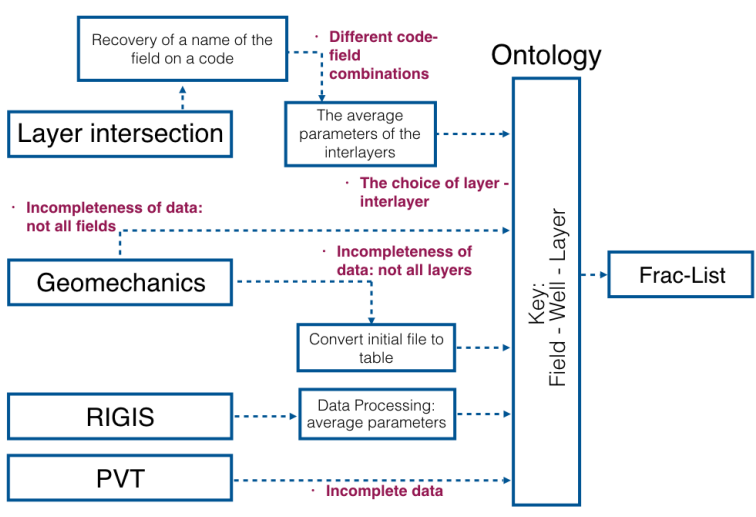

Figure 5: Block diagram (2) of the database creation

Another rather effective method that showed significant results was clustering. The use of clustering in the context of this task was not implied by learning without a teacher, but was yet another tool for finding outliers in a more complex space than two-dimensional. Also, it allowed to rely on the likely improvement in the quality of the prediction using filling in the gaps through the cluster average.

Clustering was carried out using the Density-based spatial clustering of applications with noise (DBSCAN) algorithm, because it did not require an a priori number of clusters to be specified in advance, was able to find clusters of arbitrary shape, even completely surrounded, but not connected, but most importantly, it was quite resistant to outliers.

Also, another method that eliminated more than a hundred questionable values was the Isolation Forest method, which was a variation of a random forest. The idea of the algorithm was that outliers would fall into the leaves in the early stages of tree branching and can be isolated from the main sample.

\subsection{Dimensionless parameters}

Dimensionless parameters are created to reduce the complexity and dimension of the problem (Table 3 ). Also, all the parameters have physical meaning, thus ensuring easy interpretation of the results. Here we will comment on selected parameters.

$\widetilde{V}$ is the dimensionless gel pump parameter. Its interpretation is the effective coefficient of the fracture length from the transition of different thicknesses and different stress contrasts. $\gamma$ is dimensionless effective viscosity, the ratio of viscosity to fracture forces and stress contrasts. The third parameter is the concentration of the injected proppant. $\widetilde{c}$ is the dimensionless leak-off parameter. The fifth parameter is the proppant settling parameter. $C_{f d}-$ the fracture conductivity. $\frac{d}{w}-$ bridging parameter. The parameters mentioned above can be optimized as one can change them at the stage of executing the treatment. In contrast, reservoir parameters are obviously unchangeable.

The next parameter is the ratio of the reservoir height (productive layer thickness) to the spacing between the fractures. $t_{D}$ - characteristic time scale, which gives an idea of the reservoir flow regime. The thirteenth parameter is the coefficient of evaporation. These parameters define an environment of the reservoir and are unchangeable. The last parameter is our dimensionless target function (nondimensional production) derived from the Dupuit formula.

\section{Methodology}

\subsection{Forward problem}

Once the database is created, several ML models were chosen to predict cumulative 12 months oil production. The target function is replotted in logarithmic scale to make its distribution normal (Fig. 6).

After clustering, ML regression algorithms are used: linear regression, SVM, ANN, Random Forest, Decision Trees, ExtraTrees, CatBoost, LGBM, XGBoost.

Each model was trained on the train subsample with cross validation on 5 folds. Then, models were tested on a separate test sample. Most of the ML models are decision tree based models. They have important advantages: these are fast, able to work with any number of objects and features, insusceptible to NaNs, have a small number of hyperparameters, and can work with categorical features.

Each experiment was conducted two times:

- for the entire dataset containing information about 3110 wells, and hyperparameters of the regression algorithms set to their default values.

- for wells lie in one field with default hyperparameters

The reason to conduct different experiments was to see if more homogeneous data set enhances the model's performance.

Then, we took the best performing methods based on the $R^{2}$ on test set of each experiment and combined it into ensemble to further improve the results.

After that, feature importance analysis was performed with Boruta and Sobol methods for the ensemble of the best algorithms.

Finally, uncertainty quantification was done for the model metric (the determination coefficient $R^{2}$ ) by running the model multiple times for different bootstrapped samples. The result then would have a representation of, for instance, a $95 \%$ likelihood of $R^{2}$ between $70 \%$ and $75 \%$. The scheme of the forward problem methodology is depicted on Fig. 8.

\subsection{Inverse problem}

As soon as the results of the forward problem are satisfactory, the inverse problem is formulated and solved using the reinforcement learning for dimensionless parameters. As previously mentioned, the reason we chose dimensionless parameters for the inverse problem was to reduce the complexity and dimension of the problem. For a reinforcement learning (RL) algorithm, it was necessary to determine its actions. In our case, action "zero" reduced the considered parameter of the object by its standard deviation throughout the sample. If the parameter was numerically less than zero, we set the optimized parameter to zero. Action "one" was the opposite of the action "zero": 

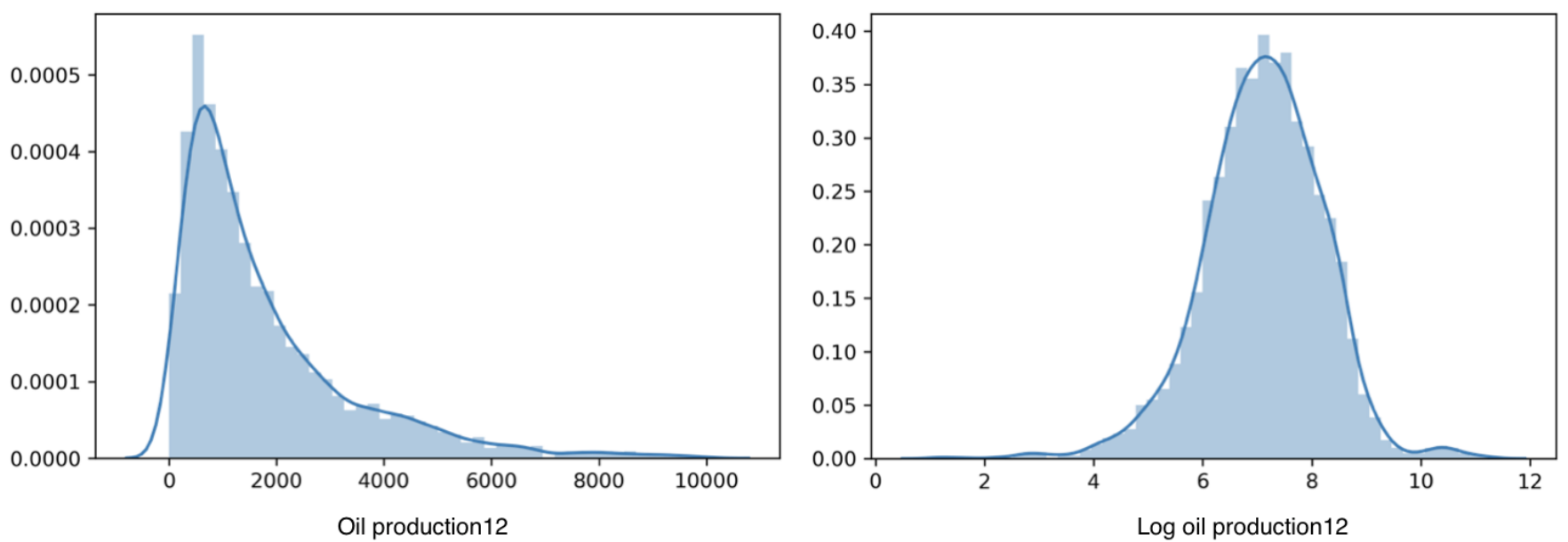

Figure 6: Distribution of the target function

the algorithm increased the parameter by its standard deviation. Action "two" was purely technical and indicated that if the set of actions from the previous iteration did not change, then the next parameter was switched. In addition, a reward function had to be defined, which is the difference between predicted label $\hat{y}$ and true target $y$ :

$$
R=\hat{y}-y
$$

The procedure of the method is illustrate in Fig. 7:

First, immutable (unchangeable) dimensionless parameters associated with geomechanics, PVT and others are given to the algorithm. Then, the algorithm randomly initializes a parameter to be optimized. The RL performs an action ("zero", "one", or "two") on the parameter. The cycle/learning repeats for one parameter until the action "two" is chosen. Third, the semioptimized parameters and immutable parameters go to the machine learning (ML) algorithm where the dimensionless target is predicted. If the reward $R$ is negative, the model is punished. During the cycle, the RL learns about states and adapts its performance to the problem, like a production stimulation engineer learns through trial and error on fracturing designs for new wells.

As soon as the maximum $R$ is reached, the optimized parameters plus parameters of the environment are given to the $\mathrm{ML}$ algorithm again. Finally, the maximum dimensionless target is predicted.

The reinforcement learning algorithm itself is based on dueling double DQN agent - a value-based reinforcement learning agent that trains a critic to estimate the return or future rewards. DQN is a variant of Q-learning. Since reinforcement learning is a relatively new area of machine learning, dueling double DQN is chosen since it is considered as the current state-of-the-art.

In addition, limits of the parameters to optimized are determined by using Gaussian Mixtures Models clustering. Figure 9 shows cluster of the dimensionless parameters on tSNE plot. As it can be seen, there are 5 clusters, where each of them have its own minimum and maximum values for dimensionless parameters.

\section{Results and Discussion}

\subsection{Clustering}

In the case of clustering there is a rule of thumb saying that a stable grouping should be maintained when a clustering method changes: for example, if the results of a hierarchical cluster analysis coincide for more than $70 \%$ of data points with results of the grouping by k-means, the assumption of stability is accepted. At the moment, in [59] there are many methods and criteria for assessing the quality of clustering results. We used the optimal number of clusters according to estimates of various indices and determined that the optimum is equal to 25 clusters. Since this work involves only identifying a small group of "similar" wells, even a rough estimate is satisfactory. However, as mentioned in the book, there are no right or wrong clustering results, because, by definition, it is an unsupervised learning method [60].

Thus, it was possible to allocate 150 wells, where about $90 \%$ of them are of the same field.

Figure 10 illustrates the clustering results using the tSNE [61] technique, which is a popular technique for non-linear dimension reduction [62], and is very convenient for visualizing high-dimensional data in two-dimensions.

\subsection{Regression}

The results are shown in the table 4 , in the figure 11 , and on the regression plot 12 for better visualization.

We can see that

- on the entire dataset the predictive accuracy is $95 \%$ on test sample reached by LGBM model. The reason of getting such a high score was due to excluding unnecessary referencing features as well as filling missing values. Before removing referencing features, the $R^{2}$ was just $63 \%$, indicating that the excluded parameters were noisy. On the other hand, filling NaNs by the average of i-th parameter clustered by well pad would create artificial data. Nevertheless, most of the wells had similar fracturing design 


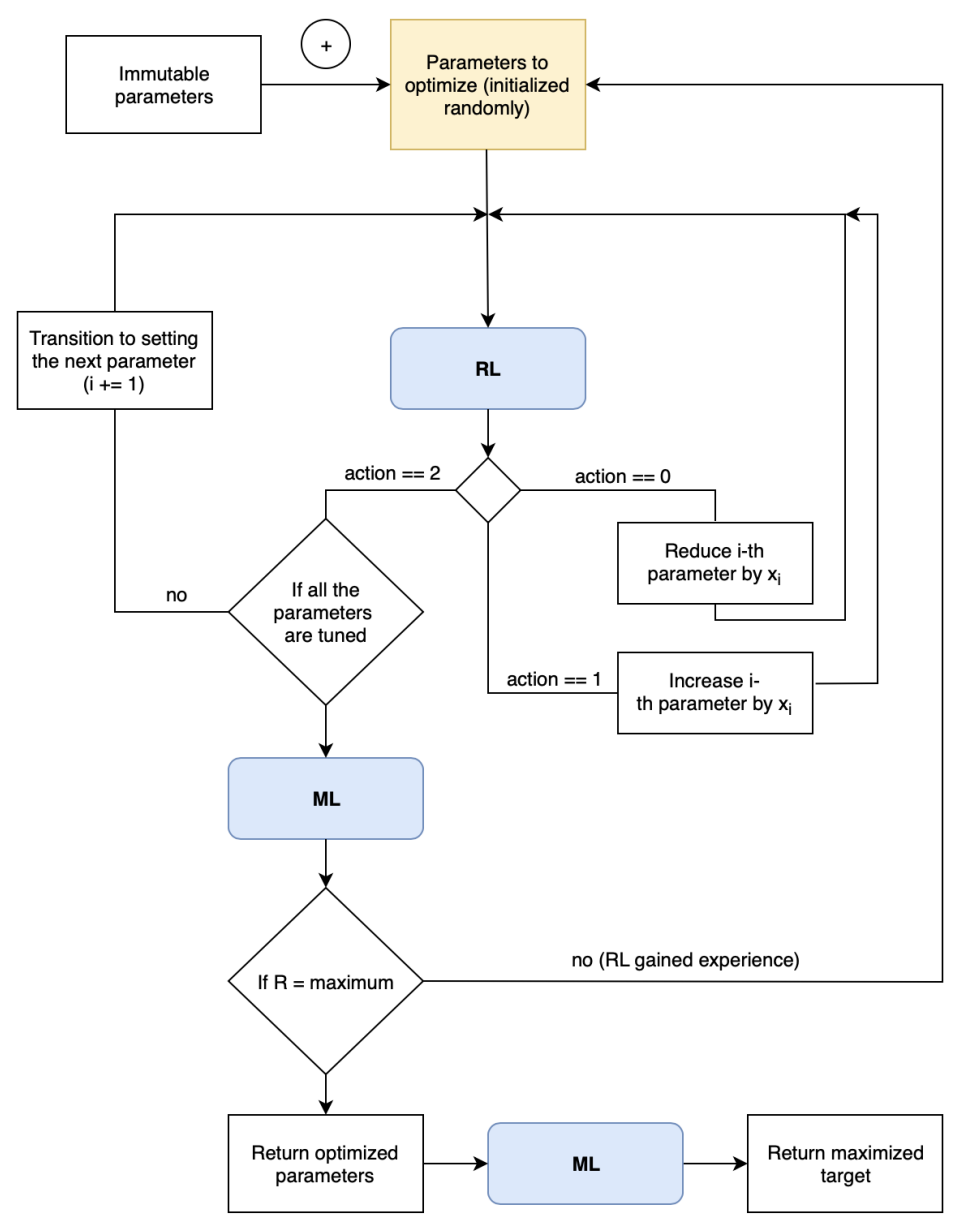

Figure 7: Design optimization algorithm

among each other, and filling missing data did not significantly change the original dataset.

- on the small dataset of 150 wells, the $R^{2}$ on test reached slightly higher score of $96 \%$. This may suggest that the model could recognize data patterns on as small as dataset with 150 samples. Hence, it is probable that our dataset contains wells with similar fracturing designs and environments.

\subsection{Ensemble of models}

As you can see, RandomForest, LGBM and XGBoost have very similar results on test sample for the entire database $(92.2 \%, 95.0 \%, 93.0 \%$, respectively). It makes sense to combine three strong algorithms into one powerful ensemble and reduce the variance of the error individually. To do this, we use stacking, since it allows the use of algorithms of a different nature. The result of the work of such an ensemble was a predictive ability of $95.4 \%$ on test sample, which, according to the authors of the article, is acceptable.

\subsection{Feature importance}

Figure 14 shows the results of feature importance. The top five important features with Boruta are: number of stages, formation volume factor, permeability times formation thickness, the type of layer, and bottom hole pressure. From the HF perspective, such results make sense since such features are indeed crucial for oil production. However, it should be mentioned that feature importance is interpreted from the point of view of importance for the model, and not from the point of view of the physics of processes.

\subsection{Uncertainty Quantification}

To completely describe the results, uncertainty has to be determined via confidence interval. To calculate the confidence interval for the ensemble model's $R^{2}$, bootstrap is used for 500 iterations, where each one is $75 \%$ the size of the entire database. As the result, the calculated confidence interval shows that there is a $95 \%$ likelihood that the confidence interval $94.1 \%$ and $96.6 \%$ covers the true $R^{2}$ of the model.

\subsection{Design Optimization}

Test of the method is performed on randomly choosing a well with known dimensionless parameters and dimensionless tar- 
get. As the result of applying the method, dimensionless target is numerically increased from 0.0116 to 0.0467 , which is a roughly fourfold increase (taking into account that the highest $D Y$ among the dataset is 0.083). Also, the algorithm gives recommended values of dimensionless parameters. Since the dimensionless parameters are interpretable, it is possible to find the original parameters thereafter (Table 5). As it can be seen, the parameters that are optimized the most were the parameters, which included geomechanics factors such as thickness $H$, Young's Modulus $E^{\prime}$, and stress contrast $\Delta \sigma$. It could be explained by the fact that geomechanics parameters had many missing values, which could cause such a big percentage change.

\section{Conclusions}

We presented the work on machine learning on field data for hydraulic fracturing design optimization. This study aimed at the data gathering, cleaning, systematization and preprocessing. We discussed in detail the issues that raise on the way towards constructing a field database, which integrated three major parts coming from essentially different sources: reservoir geology, hydraulic fracturing design and production rates.

The following important points should be emphasized:

- ML models completely depend on input data, its quality and preprocessing.

- Collection of field data is the most important step for an ML project aimed at an optimization of a stimulation treatment. A database, which has been properly validated, filled and verified with subject matter experts, allows making high-quality predictive models and using all the advantages of modern ML techniques.

- Manipulations with data and/or the deliberate use of small samples and complex ML models allow to achieve very high accuracy. However, the results show that this high accuracy does not always indicate model's ability to generalize the results obtained. Such high accuracy rates are typically a consequence of the overfitting.

Thus, an accurately formed, verified and validated field database on stimulation treatments may lead to the results that are not "ideal" (in terms of the determination coefficient), because of its inherent heterogeneities/ambiguities.

Speaking about the forward problem in determining the cumulative production, we integrated the most novel approaches available in machine learning nowadays by applying clustering, model ensembles, feature importance, and uncertainty quantification.

Finally, we attempted to solve the inverse problem by creating the algorithm based on reinforcement learning. The algorithm is capable to estimate parameters to be optimized to maximize a target while taking into account unchangeable parameters of the reservoir. However, further work has to be done to develop the procedure of restoring the dimensional variables from nondimensional ones.

\section{Acknowledgements}

The authors are grateful to the management of LLC "Gazpromneft-STC" for organizational and financial support of this work. The authors are particularly grateful to M.M. Khasanov, A.A. Pustovskikh, I.G. Fayzullin and A.S. Margarit for organizational support of this initiative. The help from E. Davidyuk, D. Popkov and V. Mikova in data gathering is greatfully appreciated.

We would like to state it explicitly that the models presented in this work are solely based on the field data provided by JSC Gazprom neft and we are grateful for the permission to publish.

Startup funds of Skolkovo Institute of Science and Technology are gratefully acknowledged by Prof. A.A. Osiptsov.

AAO, RFM acknowledge financial support from the Ministry of Education and Science of the Russian Federation (project No. 14.581.21.0027, unique identifier RFMEFI58117X0027).

\section{References}

[1] M. J. Economides, K. G. Nolte, et al., Reservoir stimulation, Vol. 2, Prentice Hall Englewood Cliffs, NJ, 1989.

[2] Y. He, S. Cheng, J. Qin, J. Chen, Y. Wang, N. Feng, H. Yu, et al., Successful application of well testing and electrical resistance tomography to determine production contribution of individual fracture and waterbreakthrough locations of multifractured horizontal well in changqing oil field, china, in: SPE Annual Technical Conference and Exhibition, Society of Petroleum Engineers, 2017.

[3] B. Al-Shamma, H. Nicole, P. R. Nurafza, W. C. Feng, et al., Evaluation of multi-fractured horizontal well performance: Babbage field case study, in: SPE Hydraulic Fracturing Technology Conference, Society of Petroleum Engineers, 2014.

[4] C. K. Miller, G. A. Waters, E. I. Rylander, et al., Evaluation of production $\log$ data from horizontal wells drilled in organic shales, in: North American Unconventional Gas Conference and Exhibition, Society of Petroleum Engineers, 2011.

[5] G. E. King, et al., Thirty years of gas shale fracturing: What have we learned?, in: SPE Annual Technical Conference and Exhibition, Society of Petroleum Engineers, 2010.

[6] S. Mohaghegh, R. Gaskari, M. Maysami, et al., Shale analytics: Making production and operational decisions based on facts: A case study in marcellus shale, in: SPE Hydraulic Fracturing Technology Conference and Exhibition, Society of Petroleum Engineers, 2017.

[7] S. D. Mohaghegh, O. S. Grujic, S. Zargari, A. K. Dahaghi, et al., Modeling, history matching, forecasting and analysis of shale reservoirs performance using artificial intelligence.

[8] S. Esmaili, S. D. Mohaghegh, Full field reservoir modeling of shale assets using advanced data-driven analytics, Geoscience Frontiers 7 (1) (2016) 11-20.

[9] R. Keshavarzi, R. Jahanbakhshi, et al., Real-time prediction of complex hydraulic fracture behaviour in unconventional naturally fractured reservoirs.

[10] E. V. Burnaev, P. V. Prikhod'ko, On a method for constructing ensembles of regression models, Automation and Remote Control 74 (10) (2013) 1630-1644. doi:10.1134/S0005117913100044. URL https : //doi .org/10.1134/S0005117913100044

[11] M. G. Belyaev, E. V. Burnaev, Approximation of a multidimensional dependency based on a linear expansion in a dictionary of parametric functions, Informatics and its Applications 7 (3) (2013) 114-125.

[12] E. Burnaev, P. Erofeev, The influence of parameter initialization on the training time and accuracy of a nonlinear regression model, Journal of Communications Technology and Electronics 61 (6) (2016) 646-660. doi:10.1134/S106422691606005X.

URL https ://doi .org/10.1134/S106422691606005X

[13] J. Guo, Y. Xiao, H. Zhu, A new method for fracturing wells reservoir evaluation in fractured gas reservoir, Mathematical Problems in Engineering 2014. 
[14] J. Schuetter, S. Mishra*, M. Zhong, R. LaFollette, Data analytics for production optimization in unconventional reservoirs, in: Unconventional Resources Technology Conference, San Antonio, Texas, 20-22 July 2015, Society of Exploration Geophysicists, American Association of Petroleum Geologists, Society of Petroleum Engineers, 2015, pp. 249269.

[15] J. Gao, F. You, Design and optimization of shale gas energy systems: Overview, research challenges, and future directions, Computers \& Chemical Engineering 106 (2017) 699-718.

[16] D. Poulsen, M. Soliman, et al., A procedure for optimal hydraulic fracturing treatment design.

[17] P. M. Saldungaray, T. T. Palisch, et al., Hydraulic fracture optimization in unconventional reservoirs, in: SPE Middle East unconventional gas conference and exhibition, Society of Petroleum Engineers, 2012.

[18] J. Betz, et al., Low oil prices increase value of big data in fracturing, Journal of Petroleum Technology 67 (04) (2015) 60-61.

[19] O. Awoleke, R. Lane, et al., Analysis of data from the barnett shale using conventional statistical and virtual intelligence techniques, SPE Reservoir Evaluation \& Engineering 14 (05) (2011) 544-556.

[20] E. Detournay, Mechanics of hydraulic fractures, Annual Review of Fluid Mechanics 48 (2016) 311-339.

[21] A. A. Osiptsov, Fluid mechanics of hydraulic fracturing: a review, Journal of Petroleum Science and Engineering 156 (2017) 513-535.

[22] T. Helmy, A. Fatai, K. Faisal, Hybrid computational models for the characterization of oil and gas reservoirs, Expert Systems with Applications 37 (7) (2010) 5353-5363.

[23] E. A. El-Sebakhy, T. Sheltami, S. Y. Al-Bokhitan, Y. Shaaban, P. D. Raharja, Y. Khaeruzzaman, et al., Support vector machines framework for predicting the pvt properties of crude oil systems.

[24] S. Wang, S. Chen, et al., A comprehensive evaluation of well completion and production performance in bakken shale using data-driven approaches.

[25] J. Schuetter, S. Mishra, M. Zhong, R. LaFollette, et al., A data-analytics tutorial: Building predictive models for oil production in an unconventional shale reservoir, SPE Journal.

[26] R. M. Balen, H. Mens, M. J. Economides, et al., Applications of the net present value (npv) in the optimization of hydraulic fractures, in: SPE Eastern Regional Meeting, Society of Petroleum Engineers, 1988.

[27] G. Hareland, P. Rampersad, J. Dharaphop, S. Sasnanand, et al., Hydraulic fracturing design optimization.

[28] C. Temizel, S. Purwar, A. Abdullayev, K. Urrutia, A. Tiwari, et al., Efficient use of data analytics in optimization of hydraulic fracturing in unconventional reservoirs, in: Abu Dhabi International Petroleum Exhibition and Conference, Society of Petroleum Engineers, 2015.

[29] W. Yanfang, S. Salehi, Refracture candidate selection using hybrid simulation with neural network and data analysis techniques, Journal of Petroleum Science and Engineering 123 (2014) 138-146.

[30] P. Pankaj, S. Geetan, R. MacDonald, P. Shukla, A. Sharma, S. Menasria, H. Xue, T. Judd, et al., Application of data science and machine learning for well completion optimization.

[31] R. M. Balen, H. Mens, M. J. Economides, et al., Applications of the net present value (npv) in the optimization of hydraulic fractures, in: SPE Eastern Regional Meeting, Society of Petroleum Engineers, 1988.

[32] R. Alimkhanov, I. Samoylova, et al., Application of data mining tools for analysis and prediction of hydraulic fracturing efficiency for the bv8 reservoir of the povkh oil field, in: SPE Russian Oil and Gas Exploration $\&$ Production Technical Conference and Exhibition, Society of Petroleum Engineers, 2014.

[33] M. Economides, R. Oligney, P. Valkó, Unified fracture design,(hardbound) orsa press, Houston, May.

[34] N. V. Queipo, A. J. Verde, J. Canelón, S. Pintos, Efficient global optimization for hydraulic fracturing treatment design, Journal of Petroleum Science and Engineering 35 (3-4) (2002) 151-166.

[35] M. Zoveidavianpoor, A. Samsuri, S. R. Shadizadeh, et al., Development of a fuzzy system model for candidate-well selection for hydraulic fracturing in a carbonate reservoir, in: SPE Oil and Gas India Conference and Exhibition, Society of Petroleum Engineers, 2012.

[36] M. Rahman, M. Rahman, S. Rahman, An integrated model for multiobjective design optimization of hydraulic fracturing, Journal of Petroleum Science and Engineering 31 (1) (2001) 41-62.

[37] R. N. Anderson*, B. Xie, L. Wu, A. A. Kressner, J. H. Frantz Jr, M. A.
Ockree, K. G. Brown, P. Carragher, M. A. McLane, Using machine learning to identify the highest wet gas producing mix of hydraulic fracture classes and technology improvements in the marcellus shale, in: Unconventional Resources Technology Conference, San Antonio, Texas, 1-3 August 2016, Society of Exploration Geophysicists, American Association of Petroleum Geologists, Society of Petroleum Engineers, 2016, pp. 254-266.

[38] M. Belyaev, E. Burnaev, E. Kapushev, M. Panov, P. Prikhodko, D. Vetrov, D. Yarotsky, Gtapprox: Surrogate modeling for industrial design, Advances in Engineering Software 102 (2016) 29 - 39. doi:https://doi.org/10.1016/j.advengsoft.2016.09.001.

URL http://www.sciencedirect.com/science/article/pii/S096599781

[39] A. Abdulraheem, M. Ahmed, A. Vantala, T. Parvez, et al., Prediction of rock mechanical parameters for hydrocarbon reservoirs using different artificial intelligence techniques.

[40] I. Makhotin, D. Koroteev, E. Burnaev, Gradient boosting to boost the efficiency of hydraulic fracturing, Journal of Petroleum Exploration and Production Technology (2019) 1-7.

[41] F. A. Anifowose, et al., Artificial intelligence application in reservoir characterization and modeling: whitening the black box.

[42] L. Saputelli, H. Malki, J. Canelon, M. Nikolaou, et al., A critical overview of artificial neural network applications in the context of continuous oil field optimization, in: SPE annual technical conference and exhibition, Society of Petroleum Engineers, 2002.

[43] S. D. Mohaghegh, A. Popa, R. Gaskari, S. Ameri, S. Wolhart, et al., Identification of successful practices in hydraulic fracturing using intelligent data mining tools; application to the codell formation in the dj-basin, in: SPE Annual Technical Conference and Exhibition, Society of Petroleum Engineers, 2002.

[44] D. M. Hawkins, The problem of overfitting, Journal of chemical information and computer sciences 44 (1) (2004) 1-12.

[45] L. Baumes, J. Serra, P. Serna, A. Corma, Support vector machines for predictive modeling in heterogeneous catalysis: a comprehensive introduction and overfitting investigation based on two real applications, Journal of combinatorial chemistry 8 (4) (2006) 583-596.

[46] E. Lolon, K. Hamidieh, L. Weijers, M. Mayerhofer, H. Melcher, O. Oduba, et al., Evaluating the relationship between well parameters and production using multivariate statistical models: a middle bakken and three forks case history, in: SPE Hydraulic Fracturing Technology Conference, Society of Petroleum Engineers, 2016.

[47] Z. Xiaofeng, A. Zolotukhin, A. Guangliang, et al., A post-fracturing evaluation method of fracture properties in multi-stage fractured wells (russian).

[48] S. Grihon, E. Burnaev, M. Belyaev, P. Prikhodko, Surrogate Modeling of Stability Constraints for Optimization of Composite Structures, Springer New York, New York, NY, 2013, pp. 359-391. doi:10.1007/978-1-4614$7551-4{ }_{1} 5$. $U R L$

\section{Appendix}

In this section we provide a table with a list of considered input features, describing a well. 


\begin{tabular}{|l|l|}
\hline (DFIT)- used Fluid 10\# or 15\# & Perforation vertical \\
\hline (DFIT)- used Fluid 20\# or 25\# & Perforations Real \\
\hline (DFIT)- used Fluid 27\# or 28\# & Period of work \\
\hline (DFIT)- used Fluid 33\# & Permability \\
\hline (DFIT)- used Fluid 35\# or 40\# & Permeability coefficient \\
\hline (DFIT)- used Fluid 40\# & Poisson's ratio \\
\hline Absolute depth & Polymer name \\
\hline Azimuth & Porosity ratio \\
\hline Bubble point pressure & Pressure bottom hole \\
\hline Buffer \% & Pressure in layer \\
\hline Clay ratio & Propant \#1 name \\
\hline Crack permeabilty & Propant \#2 name \\
\hline Crosslink concentration 1 & Propant \#3 name \\
\hline Crosslink concentration 2 & Propant \#4 name \\
\hline Crosslinker name 1 & Propant \#5 name \\
\hline Crosslinker name 2 & Proppant bottom hole \\
\hline Density of perforations & Proppant concentration in layer \\
\hline Diametr surface casing string & Proppant \#1 quantity \\
\hline Dynamic head & Proppant \#2 quantity \\
\hline Effective conductivity & Proppant \#3 quantity \\
\hline Executor & Proppant \#4 quantity \\
\hline Fluid rate & Proppant \#5 quantity \\
\hline Formation volume factor & PVT- Compressibility pore space \\
\hline Gel & PVT- Dynamic viscosity coefficient \\
\hline Gel encapsulated & PVT- Gas density \\
\hline Gel encapsulated name & PVT- Initial pressure in field \\
\hline Gel name & PVT- Plane strain modulus \\
\hline Gel other & PVT- Temperature of layer \\
\hline Gel other name & PVT- Water Compressibility \\
\hline Inclination angle & PVT- Water density \\
\hline ISIP Delta & Region \\
\hline ISIP DFIT & Stress contrast \\
\hline ISIP HF & Temperature of layer \\
\hline ISIP replacing & Tubing upper packer \\
\hline Layer & Type of geological and technical works \\
\hline Num & Used Fluid 10\# or 15\# \\
\hline Number of stages & Used Fluid 21\# or 25\# \\
\hline Oil \& gas production department & Used Fluid 33\# \\
\hline Oil saturation factor $\%$ & Used Fluid 35\# or 40\# \\
\hline Oil viscosity & Water cut \\
\hline Open end of pipe & Water density \\
\hline Packer seat & Well type \\
\hline Perforation interval & X \\
\hline & \\
\hline
\end{tabular}

Table 2: Features used to describe a well 


\begin{tabular}{|c|c|c|}
\hline$D$ & Formula & Description \\
\hline 1 & $\widetilde{V}=\frac{V_{g e l} E^{\prime}}{H^{3} \Delta \sigma}$ & Total volume of injected frac fluid \\
\hline 2 & $\gamma=\frac{K^{\prime} Q^{n} E^{2 n+1}}{H^{3 n} \Delta \sigma^{2 n+2}}$ & Effective viscosity \\
\hline 3 & $\bar{c}_{\text {prop }}=\frac{M_{\text {prop }}}{\rho_{a b s} V_{\text {gel }}}$ & Concentration of injected proppant \\
\hline 4 & $\widetilde{c}=\frac{(1-\eta) \widetilde{V}^{1 / 2}}{4 \widetilde{L} g(0)}$ & Leakoff coefficient \\
\hline 5 & $\frac{V_{g e l}}{Q H} \frac{d_{p r o p}^{1+1 / n}}{K^{\prime 1 / n}}\left[\rho_{\text {prop }}-\rho_{w}\right]^{1 / n}$ & Proppant settling velocity \\
\hline 6 & $\frac{V_{p a d}}{V_{g e l}}$ & Proppant volume \\
\hline 7 & $C_{f d} \sim \frac{K_{f}}{K_{r e s}} \frac{\Delta \sigma}{E^{\prime}}$ & Fracture conductivity (ideal case) \\
\hline 8 & $\frac{d_{\text {prop }}}{w} \sim \frac{d_{\text {prop }} E^{\prime}}{\Delta \sigma H}$ & Bridging parameter \\
\hline 9 & $\frac{H}{b_{d i s t}}$ & Spacing between fractures scaled by reservoir thickness \\
\hline 10 & $B_{g}=\frac{(V)_{p, T}}{V_{s c}}$ & Gas volume factor \\
\hline 11 & $t_{D}=\sqrt{\frac{K_{r e s} \Delta t}{\phi c_{t} \mu H^{2}}}$ & Time scale \\
\hline 12 & $\frac{\mu_{\text {oil }}}{\mu_{\text {water }}}$ & Oil-water viscosity ratio \\
\hline 13 & $\frac{P_{\text {res }}}{P_{\text {sat }}}$ & Ratio of reservoir pressure to saturation pressure \\
\hline 14 & $w=\frac{Q_{\text {water }}}{Q_{\text {fluid }}}$ & Water cut coefficient \\
\hline$D Y$ & $\frac{Q B \mu_{\text {fluid }}}{2 \pi K_{\text {res }} H \Delta P_{\text {res }}}$ & Target function (nondim. production) \\
\hline
\end{tabular}

Table 3: Dimensionless parameters: D1 - D8 - params. to optimize, D9 - D14 - res. params., DY - target

\begin{tabular}{|l|l|l|l|}
\hline & 150 wells (default hyperparam.) & 150 wells (grid search for hyperparam.) & Entire DataBase \\
\hline Linear regression & 0.659672 & 0.690023 & - \\
\hline SVM & 0.750081 & 0.789528 & - \\
\hline NN & 0.767630 & 0.907630 & - \\
\hline Random Forest & 0.511107 & 0.699967 & 0.348568 \\
\hline Decision Trees & 0.634314 & 0.836461 & 0.472548 \\
\hline ExtraTrees & 0.564013 & 0.877325 & 0.422628 \\
\hline CatBoost & 0.707260 & 0.905639 & 0.538190 \\
\hline LGBM & 0.855287 & 0.933524 & 0.531697 \\
\hline XGBoost & 0.657920 & 0.864204 & 0.522525 \\
\hline
\end{tabular}

Table 4: The result of ML algorithms 


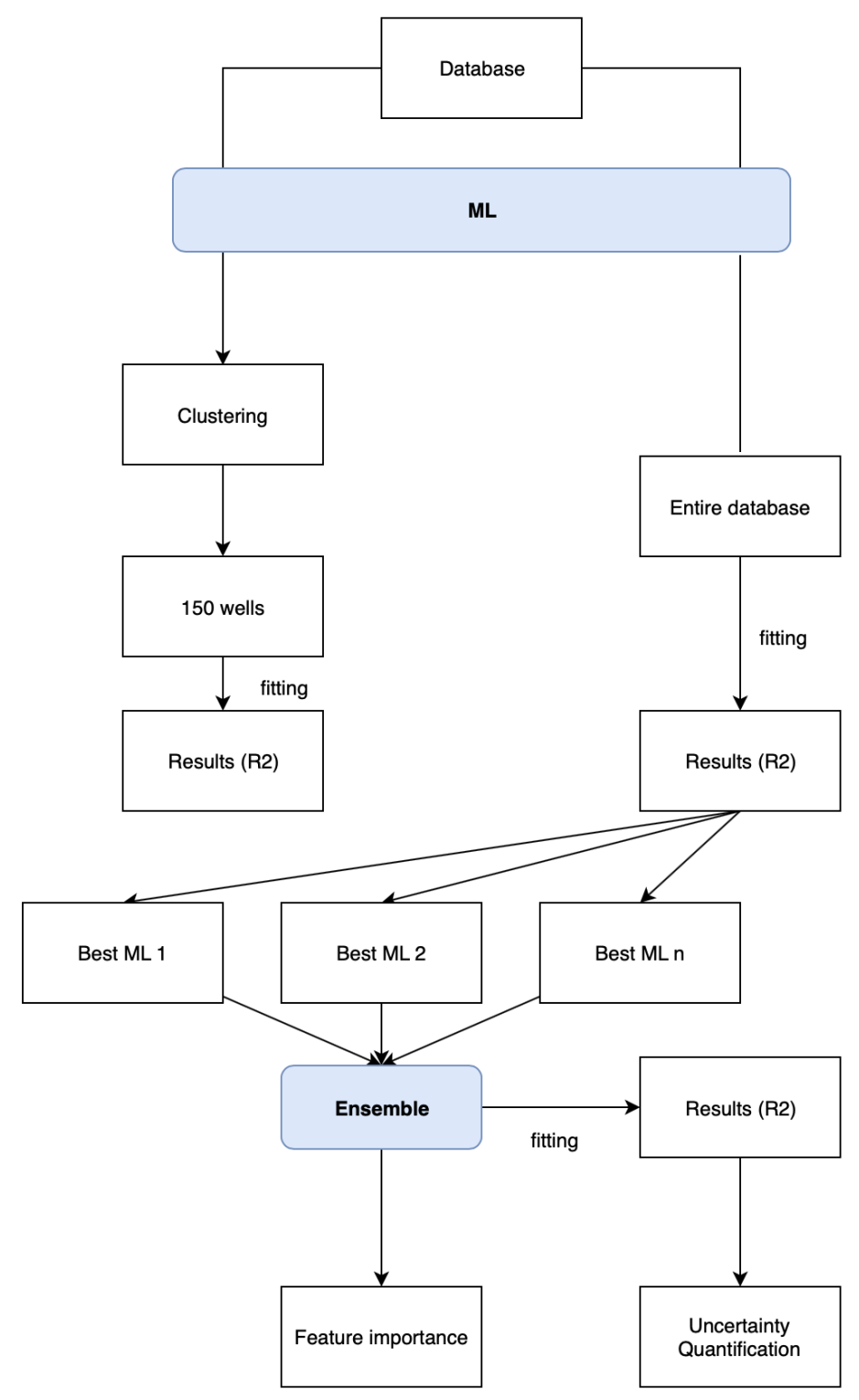

Figure 8: Forward problem approach 


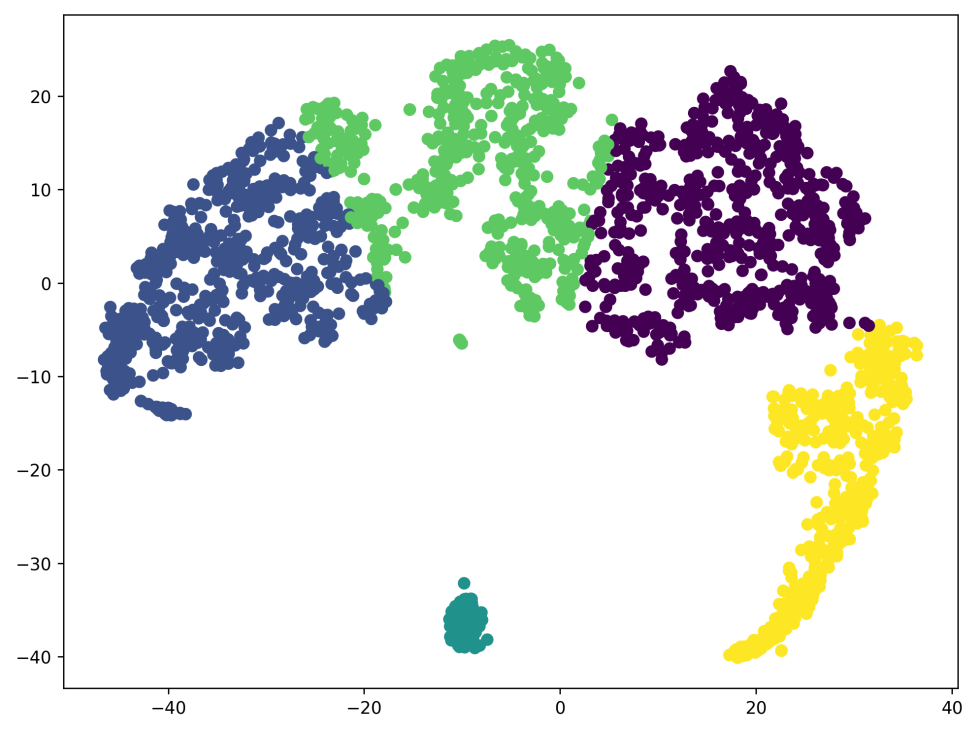

Figure 9: Clustering on dimensionless parameters

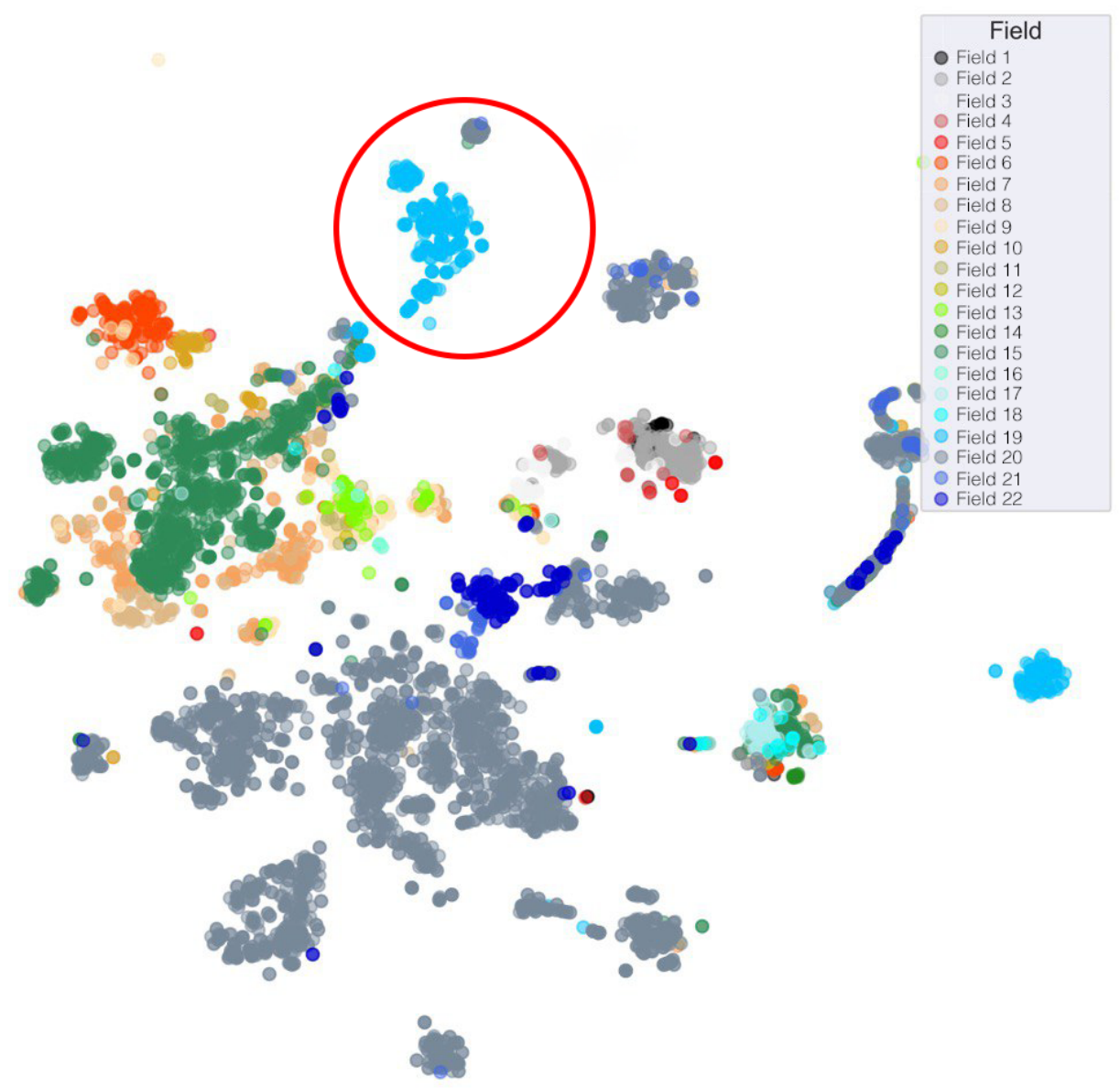

Figure 10: Application of tSNE and clustering to determine similar wells 


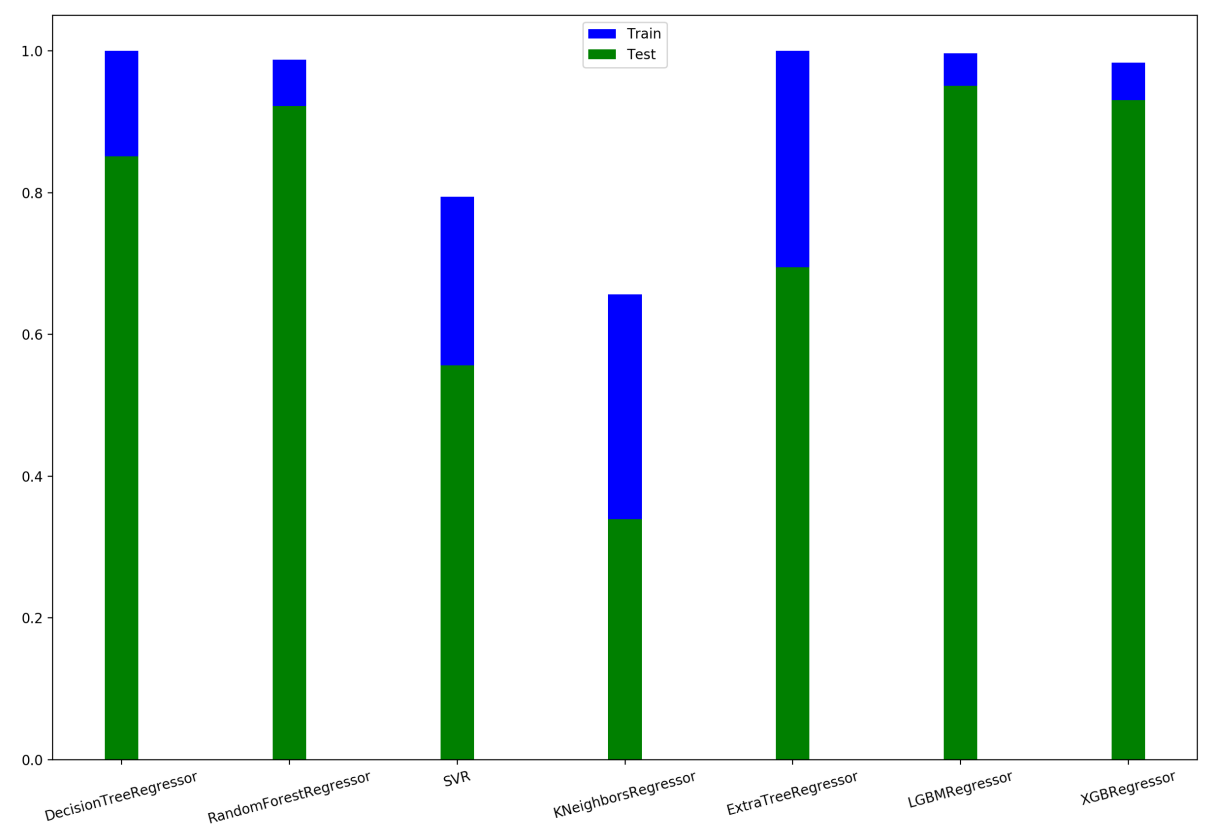

Figure 11: The $R^{2}$ results of ML algorithms on test sample for three different experiments

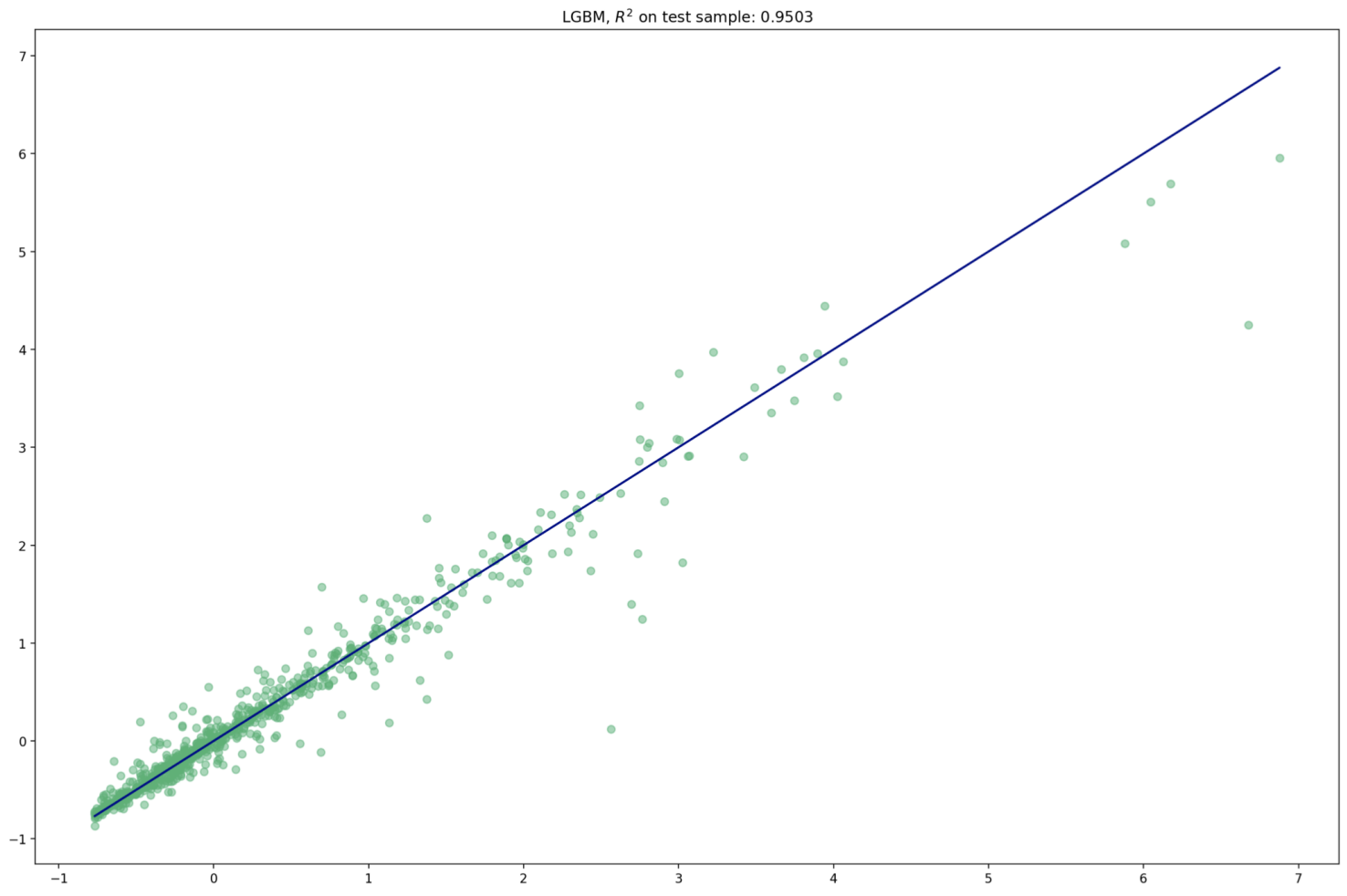

Figure 12: The regression plot for the entire database for the best performing algorithm 


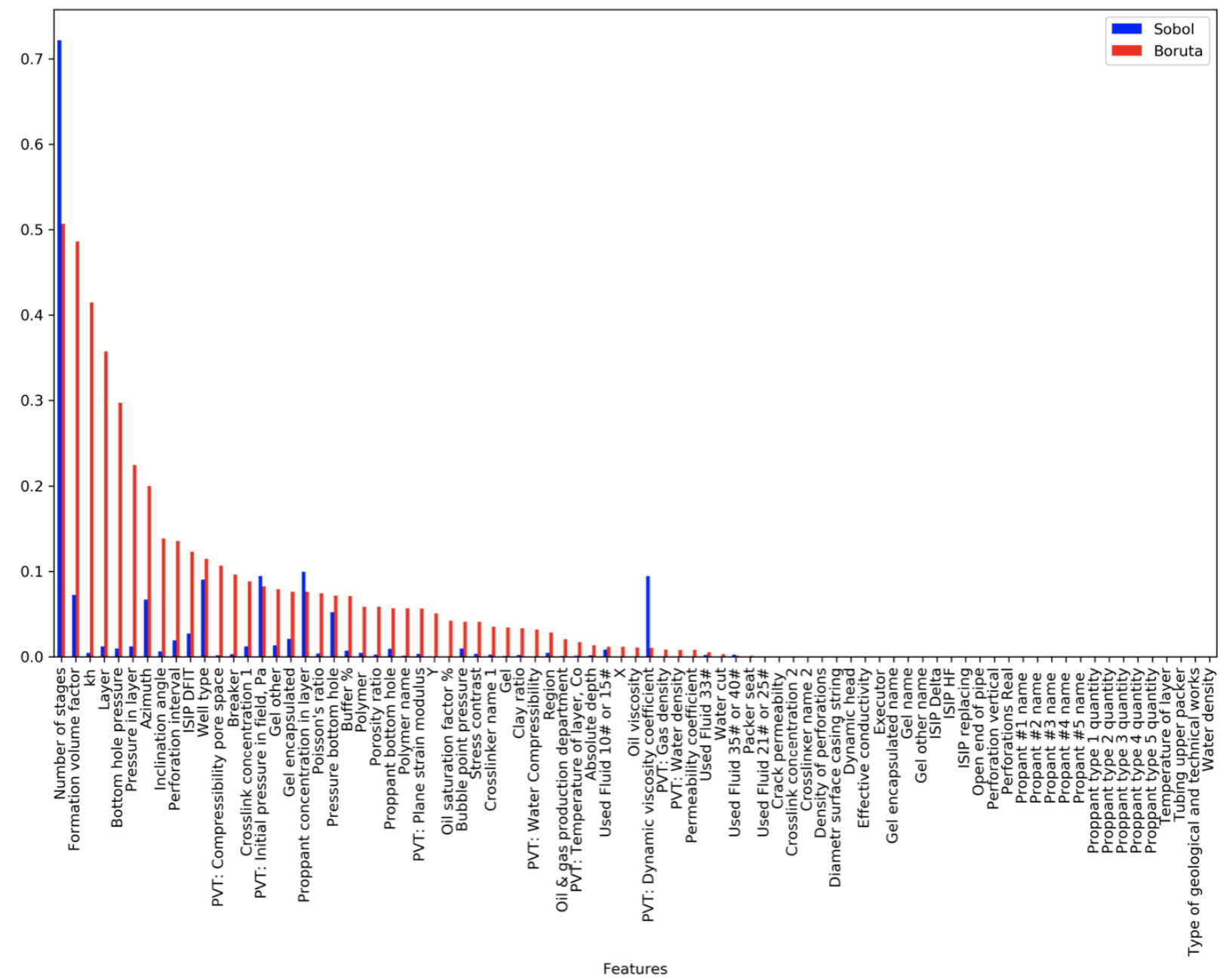

Figure 13: Feature importance of applying Boruta and Sobol methods

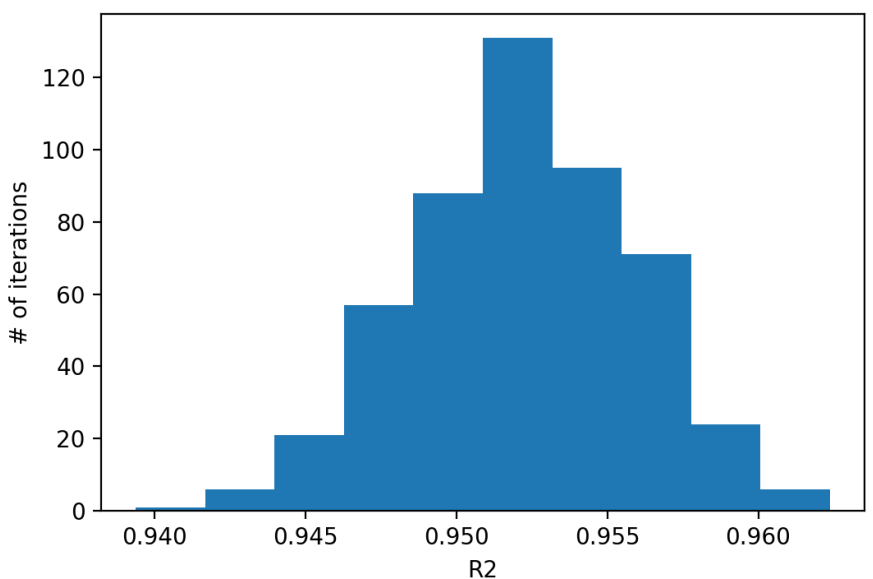

Figure 14: Confidence interval 


\begin{tabular}{|c|c|c|c|c|}
\hline$D$ & Formula & Value before opt. & Value after opt. & Percent change [\%] \\
\hline 8 & $\frac{d_{\text {prop }}}{w} \sim \frac{d_{\text {prop }} E^{\prime}}{\Delta \sigma H}$ & 9.495 & 335.8 & 97.2 \\
\hline 7 & $C_{f d} \sim \frac{K_{f}}{K_{r e s}} \frac{\Delta \sigma}{E^{\prime}}$ & 113417. & 19965. & 82.4 \\
\hline 1 & $\widetilde{V}=\frac{V_{g e l} E^{\prime}}{H^{3} \Delta \sigma}$ & 70788. & 108717. & 53.6 \\
\hline 6 & $\frac{V_{\text {pad }}}{V_{\text {gel }}}$ & 0.386 & 0.3692 & 4.28 \\
\hline 3 & $\bar{c}_{\text {prop }}=\frac{M_{\text {prop }}}{\rho_{\text {abs }} V_{\text {gel }}}$ & 0.127 & 0.123 & 3.13 \\
\hline 4 & $\widetilde{c}=\frac{(1-\eta) \widetilde{V}^{1 / 2}}{4 \widetilde{L} g(0)}$ & 41754. & 40548. & 2.89 \\
\hline 2 & $\gamma=\frac{K^{\prime} Q^{n} E^{2 n+1}}{H^{3 n} \Delta \sigma^{2 n+2}}$ & - & - & - \\
\hline 5 & $\frac{V_{\text {gel }}}{Q H} \frac{d_{\text {prop }}^{1+1 / n}}{K^{\prime 1 / n}}\left[\rho_{\text {prop }}-\rho_{w}\right]^{1 / n}$ & - & - & - \\
\hline$D Y$ & $\frac{Q B \mu_{\text {fluid }}}{2 \pi K_{\text {res }} H \Delta P_{\text {res }}}$ & 0.0116 & 0.0467 & 403 \\
\hline
\end{tabular}

Table 5: Optimization results sorted by percent change 